The distance from source to sample was $28 \mathrm{~cm}$, whereas the sample to film distance varied from 15 $\mathrm{mm}$ to $20 \mathrm{~mm}$ (the Newkirk method requires 0.1 $\mathrm{mm})$. The second slit $\left(S_{2}\right)$ was adjusted such that the small divergence of the incident beam effectively separates the $K \alpha_{1} \alpha_{2}$ doublet, allowing only the $K \alpha_{1}$ component to diffract. When the line of $x$ rays impinge on the sample, a line element of volume diffracts and its image is registered on the photographic plate. The resulting image reveals a projection of the flaws in the irradiated volume. The total image is the superposition of these line elements formed during translation.

Characteristic copper radiation from a spot-focus $\mathrm{x}$-ray tube was employed in this investigation. The effective size of the spot at $6^{\circ}$ was $0.4 \mathrm{~mm} \times 0.8 \mathrm{~mm}$. Nickel foil $(\approx 0.002$-in.) was placed over the film holder to reduce background scatter. Kodak type- $A$ plates were used; the exposure time varied from 1 to $3 \mathrm{hr}$.

A polished ${ }^{8}$ (111) GaAs substrate slice was examined with the scanning-reflection method; a (404) topograph (Fig. 2) reveals numerous polishing scratches. ${ }^{9}$ Under optical examination, the polished surface appeared to be damage-free. A region of macromisorientation was also observed in the topographic image; visual examination yielded no evi- dence of this gross defect. A (242) topograph (Fig. 2) of the subsequently deposited GaAs epitaxial film exposes many localized surface protrusions (hillocks) which exclude the material from further processing. A prolonged clean-up etch ${ }^{10}$ before deposition reduced the number of hillocks on later deposits. In this case the existence of hillocks is attributed to the deformed layer at the substrate. Inclusions at the substrate-film interface have also activated these defects. ${ }^{11}$

\footnotetext{
${ }^{1}$ A. R. Lang, J. Appl. Phys. 29, 597 (1958).

${ }^{2}$ G. H. Schwuttke, J. Appl. Phys. 33, 2760 (1962).

${ }^{3}$ J. B. Newkirk, J. Appl. Phys. 29, 995 (1958).

${ }^{4}$ M. Renninger, Phys. Rev. Letters 1, 104 (1962).

${ }^{5} \mathrm{~J}$. K. Howard and R. H. Cox, to be published.

"Research and Investigation of Gallium Arsenide-Indium Arsenide Alloy Systems," Contract AF 33(615)-1272, Final Report, June 1965.

"Development of Gallium Arsenide-Phosphide Graded BandGap Base Transistor Structures," Contract No. NObsr 91238 , Second Interim Engineering Report, 23 September-23 December 1964, p. 16.

${ }^{8}$ A. Reisman and R. Rohr, J. Electrochem. Soc. 111,1425 (1964).

${ }^{9}$ The polishing scratches probably originated from GaAs chips imbedded in the polishing pad.

${ }^{10}$ J. L. Richards and A. J. Crocker, J. Appl. Phys. 31, 611 (1960).

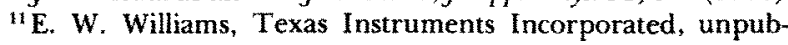
lished information.
}

\section{HOLOGRAMS ON THICK EMULSIONS}

(effect of emulsion thickness; 2-beam interferometry; E)

\author{
Albert A. Friesem \\ Institute of Science and Technology \\ The University of Michigan \\ Ann Arbor, Michigan 48107 \\ (Received 15 July 1965)
}

In the wavefront reconstruction process of Gabor," the use of an off-axis reference beam for separating the real and virtual images, as well as for separating various noise components from these reconstructed images, has been demonstrated. ${ }^{2,3}$ The theory behind the technique treats the recording film emulsion as a two-dimensional medium. The case where the recording medium is three dimensional has been treated by Denisyuk ${ }^{4}$ and by van Heerden.5 Here, the interference effects are recorded as surfaces within the recording medium. The diffraction process obeys the law of Bragg reflections, analogous to diffraction in a crystal, each diffracted order being produced only for a given orientation of the hologram. The generation of the diffracted orders that produce the virtual and the real image occurs at different orientations of the hologram.

In our laboratory, holograms have been made on many different emulsions, with Kodak type-649 emulsion producing the most interesting holograms. This emulsion has a thickness of about $6 \mu$ after developing. When the recorded fringes have a spacing comparable to or less than the emulsion thickness, the emulsion acts as a three-dimensional medium. As our hologram tehniques have become more so- 
phisticated, the fringe frequencies have increased, fringe frequencies of $1000 \mathrm{l} / \mathrm{mm}$ now being common. At these fringe frequencies, the Bragg reflection characteristics become quite prominent.

Holograms were constructed taking advantage of the three-dimensional effect, using the configuration shown in Fig. 1. This is an adaptation of a method used earlier, ${ }^{3}$ except that three beams are employed. A monochromatic, spatially coherent source illuminates objects $A$ and $B$. The scattered waves from these objects interfere with the reference beam, which is reflected from the mirror, and the resulting interference pattern is then recorded on the hologram.

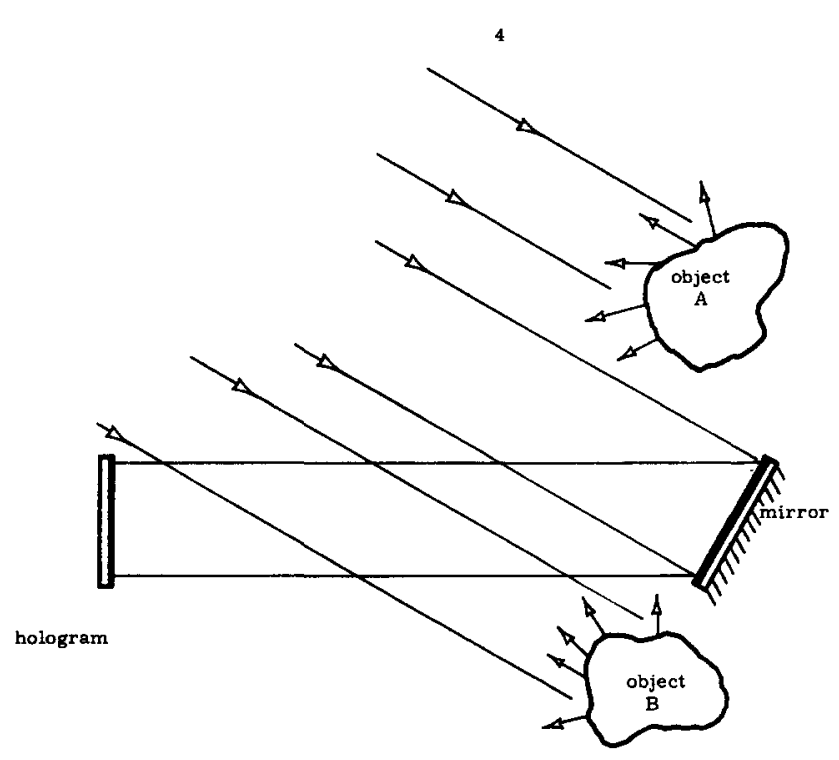

Fig. 1. System used for making the hologram.
The objects were arranged so that the angles subtended between either object and the reference beam were the same. Thus, if the emulsion thickness is assumed to be negligible, as is normally done, then in the reconstruction the real image of object $A$ would fall on the virtual image of object $B$ and the real image of object $B$ would fall on the virtual image of object $A$, thereby degrading all reconstructed images.

In the actual reconstruction, however, the virtual images of both objects could be observed visually, without any trace of the real images. Rotation of the plate caused the virtual images to fade and then disappear, and the real images to appear in their place. Another interesting observation was made when the hologram was rotated by approximately $180^{\circ}$ and so oriented as to optimize the intensity of the virtual image of $A$. For this orientation, only $A$ could be observed, and further rotation was required before the image $B$ came into view, whereupon the image $A$ disappeared.

In order for the overlapping real and virtual images to be separated, the angle between objects and reference beam had to be sufficiently great that the interference fringes thus generated had spacings of about $6 \mu$ or less thus satisfying the previously stated criterion.

The author expresses his gratitude to $\mathrm{E}$. N. Leith for his helpful comments and encouragement.

'D. Gabor, Proc. Roy. Soc. (London) A197, 454 (1949).

${ }^{2}$ E. N. Leith and J. Upatnieks, J. Opt. Soc. Am. 52, 1123 (1962).

${ }^{3}$ E. N. Leith and J. Upatnieks, J. Opt. Soc. Am. 54, 1295 (1964).

${ }^{4}$ Y. N. Denisyuk, Optics and Spectroscopy 15, 279 (1963).

${ }^{5}$ P. J. van Heerden, Appl. Opt. 2, 393 (1963).

\section{UV LASER EMISSION BY GRYSTAL EXCITONS}

$\left(\mathrm{KBr} ; 3150 \AA ; 7^{\circ} \mathrm{K} ; \mathrm{E} / \mathrm{T}\right)$
E. L Fink

General Dynamics/Pomona Physics and Infrared Section Pomona, California

(Received 14 June 1965)
This Letter reports the results of experimental studies which have been conducted on the stimulated emission of ultraviolet radiation in optically pumped potassium bromide single crystals. It is concluded that the observed laser emission is consistent with an exciton scheme of population inversion devised for the alkali halides.

The high radiative quantum efficiency (at low temperatures), Stokes shift and strong absorption characteristics occurring in the exciton radiative 